\title{
Diversidad y grupos funcionales de insectos en cultivos de arroz y sus bordes bajo manejo convencional y agroecológico en Santa Fe, Argentina
}

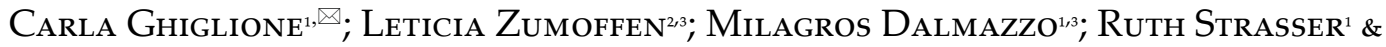 \\ AndrÉs M. ATtADEMO ${ }^{3 / 4}$
}

${ }^{1}$ Cátedra de Entomología, Facultad de Humanidades y Ciencias, Universidad Nacional del Litoral. Provincia de Santa Fe, Argentina. ${ }^{2}$ INTA Ángel Gallardo. Provincia de Santa Fe, Argentina. ${ }^{3}$ CONICET, Argentina. ${ }^{4}$ Laboratorio de Ecotoxicología, Facultad de Bioquímica y Ciencias Biológicas, Universidad Nacional del Litoral. Provincia de Santa Fe, Argentina.

\begin{abstract}
Resumen. Los servicios ecosistémicos que generan los insectos, tales como el control de plagas y la polinización, son fundamentales para la producción y la sanidad agrícola de los alimentos. Los objetivos del presente trabajo fueron 1) comparar la diversidad de insectos que presentan los cultivos de arroz y sus taipas (bordes o albardones) en dos establecimientos bajo dos condiciones de manejo (agroecológico y convencional), 2) caracterizar grupos funcionales de insectos en cultivos de arroz y sus taipas, con diferente tipo de manejo, y 3 ) analizar las interacciones de los grupos funcionales de insectos con la vegetación de las taipas. Los muestreos se realizaron durante un ciclo de producción de arroz en la primavera-verano 2017-2018, en establecimientos con prácticas de manejo convencional (EMC) y agroecológico (EMA), en una zona de producción arrocera intensa (Departamento San Javier, Santa Fe, Argentina). Se capturaron 1008 insectos pertenecientes a 237 especies y morfoespecies mediante el uso de redes entomológicas. Las taipas mostraron mayor riqueza, abundancia y diversidad que los cultivos, y el EMA se destacó por la presencia de los cinco grupos funcionales. En ambos establecimientos se capturaron Sepedonea sp., Protodictya sp. y Eryopis connexa (L.), conocidos controladores de gasterópodos e insectos plagas. Se registraron 397 interacciones planta-visitante floral en las taipas agroecológicas y sólo 175 en taipas convencionales. Los resultados obtenidos en este trabajo constituyen un aporte novedoso al conocimiento de los insectos presentes en las taipas y en el cultivo de arroz, y aportan datos de diversidad, composición y distribución de la comunidad de insectos, como así también de la oferta floral de la vegetación que crece en taipas y su relación con la entomofauna.
\end{abstract}

[Palabras clave: servicios ecosistémicos, interacciones planta-visitante floral, depredadores, parasitoides, taipas]

Aвstract. Diversity and functional groups of insects in rice crops under conventional and agroecological management in Santa Fe province, Argentina. Ecosystem services provided by insects, such as pollination and pest control, are essential for food production and agricultural health. The aims of this work were to 1) compare insect diversity in rice crops and their containment edges in two farms under different management systems (conventional and agroecological), 2) characterize functional groups of insects in rice crops and their containment edges, under different management systems, and 3) analyze the interactions of functional groups of insects with vegetation in the containment edges. Sampling was conducted during a rice production cycle in the spring-summer 2017-2018 in farms under conventional management practices (EMC) and agroecological management (EMA) in an area of intensive rice production (San Javier Department, Santa Fe, Argentina). A total of 1008 insects belonging to 236 species and morphospecies were captured using entomological nets. Containment edges exhibited higher richness, abundance and diversity than crops, with the EMA standing out for the presence of all five functional groups. On both farms, Sepedonea sp., Protodictya sp. and Eryopis connexa (L.), known control agents of gastropods and insect pests, were recorded. A total of 397 insect-plant interactions were registered in agroecological containment edges, and only 175 in conventional ones. The results obtained in this work make a novel contribution to the knowledge of insects present in containment edges and in rice crops. This work provides knowledge on the diversity, composition and distribution of the insect community, as well as data on the floral offer of the vegetation growing in containment edges and its relationship with the entomofauna.

[Keywords: ecosystem services, plant-insect interactions, predators, parasitoids, containment edges]

Editor asociado: Fernando Milesi

ghiglionecarla0@gmail.com 


\section{INTRODUCCIÓN}

La agricultura es la base del sustento alimentario del hombre y depende de procesos naturales, denominados servicios ecosistémicos, brindados por la biodiversidad (IPBES-7 2019). Los servicios ecosistémicos son fenómenos estrictamente ecológicos que resultan en algún beneficio para los humanos de manera directa o indirecta, vinculados a la estructura, los procesos y las funciones de los ecosistemas (Fisher and Turner 2008). La expansión y la intensificación de la agricultura provoca la pérdida de biodiversidad en los ecosistemas (Tscharntke et al. 2005; Medan et al. 2011) y altera la provisión de servicios ecológicos que regulan el flujo de energía y materiales en el ambiente (Chapin et al. 2000). En este sentido, la conversión de ambientes naturales en agroecosistemas ha provocado cambios a escala global, regional y local en la composición, la abundancia y la diversidad de especies (Tylianakis et al. 2007; Medan et al. 2011; Molina et al. 2014). Estos cambios contribuyen a la homogeneización de los ambientes a través de la colonización de especies no-nativas y la extinción de las nativas, mientras que la eliminación de la vegetación nativa es la principal causa de pérdida de biodiversidad (Medan et al. 2011; Marrero et al. 2014).

En los agroecosistemas, el componente natural y semi-natural está representado por fragmentos incluidos en una matriz de suelo destinado a la agricultura. Estos remanentes naturales proporcionan heterogeneidad al ambiente, con condiciones más estables y mayor diversidad y complejidad en la estructura de la comunidad vegetal (Poggio et al. 2010, 2013; Molina et al. 2016), actuando como refugio para las especies tanto a escala local como de paisaje (Medan el al. 2011; Molina et al. 2014). La comunidad vegetal de estos ambientes es la base de las tramas tróficas, ya que mantienen poblaciones permanentes de artrópodos que sustentan niveles tróficos superiores y cumplen una función clave en la provisión de servicios ecosistémicos (Altieri and Nicholls 2007; Medan et al. 2011; Molina et al. 2014, 2016; Lindgren et al. 2018). La diversidad y la presencia de poblaciones viables de los insectos es esencial para la provisión de servicios de polinización y regulación de poblaciones de insectos plaga (Öckinger and Smith 2007; Molina et al. 2014, 2016). Si bien la presencia de bordes no cultivados dentro de los agroecosistemas y su efecto sobre las comunidades y grupos funcionales de artrópodos han sido abordados desde múltiples perspectivas en distintos monocultivos en la Argentina (Medan et al. 2011; Torretta and Poggio 2013; Marrero et al. 2014; Molina et al. 2014, 2016; Sáez et al. 2014; Le Féon et al. 2016), estas aproximaciones son escasas en cultivos de arroz, en los que predominan estudios focalizados en complejos de insectos plaga (de Remes Lenicov et al. 2014; Kruger and Burdyn 2015).

El arroz (Oryza sativa L.) es uno de los cereales de mayor producción a nivel global, y se lo considera el alimento base para la mitad de la población mundial (Pinciroli et al. 2015). Este cultivo presenta características propias que lo diferencian de otros agroecosistemas: es un hábitat sobre todo acuático, con una fase de sequía generalmente predecible, que se puede definir como un ecosistema de humedal temporal manejado de manera agronómica (Bambaradeniya and Amerasinghe 2004). Presenta dos hábitats diferentes: parcelas usualmente rectangulares, inundadas, donde crecen las plantas de arroz, y taipas (bordes elevados o albardones) que contienen y dirigen el agua, donde, en general, crece otro tipo de vegetación (Bambaradeniya 2003). Las taipas constituyen ambientes que se distribuyen dentro del cultivo, aportan heterogeneidad al agroecosistema y permiten la conexión de sus comunidades con las de los remanentes de vegetación natural (Silva et al. 2020). Debido a estas características, algunos autores consideran que los cultivos de arroz son ambientes muy estables a largo plazo, lo que favorece la viabilidad de las poblaciones de una gran variedad de organismos (Bambaradeniya 2003; Bambaradeniya and Amerasinghe 2004; Bambaradeniya and Edirisinghe 2009; Edirisinghe and Bambaradeniya 2010). A pesar de esto, la intensificación de labranza para producir arroz de alto rendimiento bajo la forma convencional implica usar fertilizantes y biocidas químicos (e.g., neonicotinoides, diamidas, piretroides, carbamatos, organofosforados y herbicidas como el glifosato) para controlar insectos perjudiciales y malezas, así como para eliminar vegetación no cultivada que crece sobre las taipas (Attademo et al. 2007, 2018; Silva et al. 2020). Por el contrario, la producción de arroz orgánica se basa en eliminar el uso de agregados químicos sintéticos en forma de fertilizantes y biocidas y promover el uso de técnicas alternativas para controlar insectos y malezas. Además, las taipas son mantenidas con vegetación espontánea, no cultivada (Silva et al. 2020). 
Estudios realizados en el sur de Brasil señalan que la diversidad y la abundancia de insectos depredadores y parasitoides registrados sobre las taipas con vegetación natural son diferentes entre cultivos de arroz orgánico y convencional debido al rol clave de la vegetación no cultivada presente en los bordes (Pires et al. 2016; Acosta et al. 2017; Silva et al. 2020). Muchos estudios sobre la relación entre biodiversidad y servicios ecosistémicos en cultivos de arroz se han enfocado en el rol de la riqueza y de la abundancia de especies de grupos funcionales (Duré et al. 2008; Pires et al. 2016; Acosta et al. 2017; Attademo et al. 2018; Silva et al. 2020). Sin embargo, las funciones ecosistémicas dentro de una comunidad frecuentemente dependen también de la identidad taxonómica de las especies y de sus interacciones (Kremen et al. 2004). Analizar redes de interacción planta-visitante floral sobre las taipas permite comparar comunidades a través de parámetros que indican tipo, frecuencia y heterogeneidad con las que ocurren las interacciones, aspectos escasamente explorados en sistemas de cultivo de arroz.

En la Argentina, el cultivo de arroz se desarrolla en la Región Litoral, en particular en las provincias de Santa Fe, Entre Ríos, Corrientes y Formosa ( $90 \%$ de la producción nacional; en la campaña 2018-19 se alcanzaron más de 1.1 millones de toneladas, con 190800 ha sembradas [SIIA 2019]). En la provincia de Santa Fe se cultivaron 29760 ha de arroz en la campaña 2017-18 (SEA 2017), de las cuales sólo 10 ha se conocen como destinadas a la producción con manejo de base agroecológica. Estas producciones reproducen las prácticas de la agricultura orgánica, pero constituyen una alternativa para pequeños y medianos productores que por diferentes motivos (e.g., producción familiar y autoconsumo, comercialización en mercados no formales, discontinuidad en la producción, limitaciones económicas impuestas por altos costos de la certificación, entre otras) no pueden acceder a la certificación de producción orgánica oficial (Attademo observación personal). A pesar de la amplia superficie destinada para este cultivo en la provincia, son escasos los trabajos que evalúan la biodiversidad bajo distintos tipos de manejo (Attademo et al. 2018; Lorenzón et al. 2020). También se desconoce el rol de la vegetación natural en las taipas como sustento degrupos funcionales de insectos. Los objetivos del presente trabajo fueron 1) comparar la diversidad de insectos que presentan los cultivos de arroz y sus taipas en dos establecimientos bajo condiciones de manejo diferentes (agroecológico y convencional), 2) caracterizar grupos funcionales de insectos en cultivos de arroz y sus taipas con diferente tipo de manejo, y 3) analizar las interacciones de los grupos funcionales de insectos con la vegetación de las taipas.

\section{Materiales y Métodos}

\section{Sitio de estudio}

El área de estudio está ubicada en el centroeste de la provincia de Santa Fe (Argentina), en el departamento de San Javier (Figura 1), en una zona de producción arrocera intensa (SEA 2017). Fitogeográficamente, pertenece a la ecorregión del Espinal, caracterizada por bosques bajos de especies leñosas xerófilas donde predominan bosques de ñandubay (Prosopis affinis Spreng.), algarrobo negro (Prosopis nigra [Griseb.] Hieron.) y espinillo (Vachellia caven [Molina] Seigler and Ebinger), entre otros (Burkart et al. 1999). Los dos establecimientos seleccionados para este estudio (Figura 1a) son adyacentes a ambientes naturales sujetos al pastoreo por ganado, con bosques xerófilos de la ecorregión del Espinal en elevaciones más altas, y pantanos y praderas inundadas en las bajas (LópezLanús et al. 2010).

En la región, los establecimientos productores de arroz que presentan manejo convencional (como el seleccionado para este trabajo: EMC: $30.596^{\circ} \mathrm{S}-60.108^{\circ} \mathrm{O}$ ), incluyen la aplicación aérea antes de sembrar de herbicidas como glifosato para el control de pastos; de fungicidas (bentazón y clomazone), generalmente mezclados con otros pesticidas; de organofosforados (clorpirifós $500 \mathrm{~cm}^{3} / \mathrm{ha}$ ) e insecticidas piretroides (lambda-cihalotrina $100 \mathrm{~cm}^{3} / 20 \mathrm{~L}$ ) durante la floración para controlar artrópodos, y la fertilización cuando las plantas de arroz tienen entre 7 y 10 días (Romero et al. 2009; Attademo et al. 2014, 2015). Fuera de la temporada del cultivo (marzo-noviembre) se utiliza el sistema de barbecho. Las fechas de muestreo de insectos no se solaparon con las aplicaciones de agroquímicos (los muestreos fueron al menos 10 días después).

La producción de arroz con manejo agroecológico (EMA: $30.608^{\circ} \mathrm{S}-59.965^{\circ} \mathrm{O}$ ) ocurre en un establecimiento de 10 ha. Este tipo de práctica se realiza sin aplicación de agroquímicos; el control de malezas dentro del 


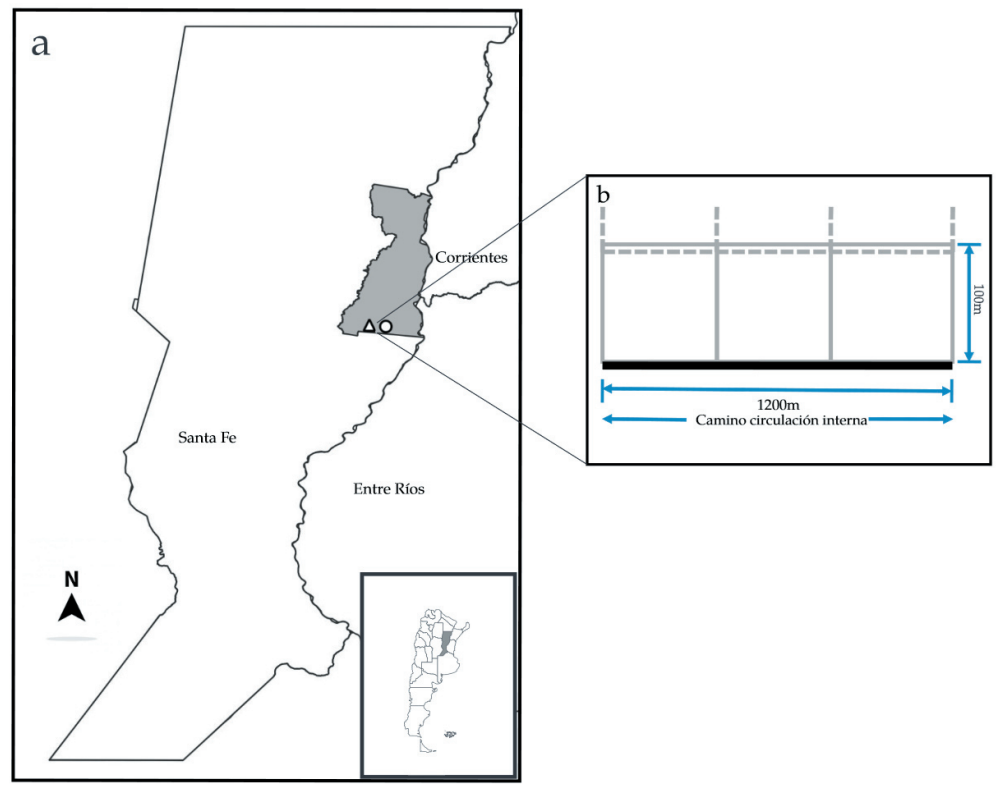

Figura 1. a) Área de estudio dentro de la provincia de Santa Fe (Argentina). El área gris indica el departamento de San Javier. El triángulo corresponde al establecimiento en que se cultiva arroz con manejo convencional (EMC) y el círculo al establecimiento con manejo agroecológico (EMA). b) Diagrama de muestreo utilizado en ambos establecimientos. Los cuadrados representan los lotes con cultivo muestreados y la línea negra indica la taipa sobre la cual se trazaron al azar las transectas de $100 \times 2 \mathrm{~m}^{2}$.

Figure 1. a) Study area in Santa Fe province (Argentina). San Javier department is indicated in grey. The farm where rice is cultivated under conventional management (EMC) is indicated with a triangle, and the farm under agroecological management (EMA) is indicated with a circle. b) Sampling diagram used for both farms. Squares represent the rice sampling plots and the black line indicates the containment edges (taipas) on which $100 \times 2-\mathrm{m}^{2}$ transects were randomly established.

cultivo se realiza de manera manual y el agua que se utiliza para inundar los lotes se filtra para evitar que entren semillas no deseadas. Fuera de la temporada de cultivo se realiza un manejo rotativo con ganado vacuno. En este sitio se han realizado estudios que confirman que no se han utilizado agroquímicos (Attademo et al. 2014, 2018).

En cada establecimiento se seleccionaron tres lotes contiguos de cultivo de arroz de 3-5 ha cada uno y su taipa con vegetación no cultivada (Figura 1b). Las taipas son albardones de $80-120 \mathrm{~cm}$ de altura, 2-3 m de ancho y 300-500 m de largo que actúan como barrera perimetral para contener el agua que inunda el cultivo de arroz. En ninguno de los dos tipos de manejo se elimina la vegetación de crecimiento espontáneo sobre las taipas. En todos los casos, las taipas seleccionadas para los muestreos presentaban de un lado el cultivo de arroz y del otro, un camino de tierra de circulación vehicular interna de cada establecimiento.

\section{Muestreos}

Todos los muestreos de insectos y sus interacciones se realizaron durante un mismo ciclo de producción primavera-verano (noviembre 2017 a marzo 2018). La colecta de insectos se realizó a lo largo de toda la fenología del cultivo, desde el estadío de plántula hasta la madurez y cosecha, sumando un total de $20 \mathrm{~h}$ por establecimiento. Estas estuvieron distribuidas en cuatro jornadas para el EMA (Oryza sativa var. jazmín) y tres jornadas para el EMC (Oryza sativa var. largo fino), ya que la variedad implantada era de ciclo corto. Todos los muestreos fueron realizados entre las 9:00 h y las 16:00 h, rotando el establecimiento por el que se comenzaba de manera de abarcar toda la franja horaria diurna en cada sitio.

En cada jornada se recorrieron 12 transectas de $100 \times 2 \mathrm{~m}^{2}$ trazadas a lo largo de las taipas en cada establecimiento. Para construir las redes de interacción planta-visitante floral, cada transecta fue recorrida por un observador que registró las especies vegetales en flor y los insectos presentes en las flores ubicadas en los parches de vegetación atravesados por las transectas ( 4-5 parches/transecta) durante $5 \mathrm{~min} /$ parche (ver Chacoff et al. 2012). Pasadas dos horas de ese primer recorrido se caminó nuevamente por cada transecta a paso constante colectando insectos con red entomológica $(40 \mathrm{~cm}$ de diámetro y 
$80 \mathrm{~cm}$ de profundidad del copo) para hacer las estimaciones de abundancia, riqueza y diversidad de insectos.

Las colectas de insectos dentro de los cultivos de arroz se hicieron de la misma manera sobre 12 transectas de $100 \times 2 \mathrm{~m}^{2}$ por establecimiento, ubicadas a $12 \mathrm{~m}$ de distancia paralelas a las taipas. Los insectos y plantas que no pudieron ser identificados en el campo fueron colectados para su determinación en laboratorio. Las plantas fueron herborizadas para su transporte y posterior identificación. Los insectos se conservaron en frío para su transporte y luego se los almacenó en el freezer hasta su preparación e identificación con lupa estereoscópica siguiendo claves dicotómicas (McAlpine et al. 1981; Morrone and Coscarón 1998; Cordo et al. 2004; Triplehorn and Johnson 2005; Fernández and Sharkey 2006; Michener 2007; Claps et al. 2008; Roig-Juñent et al. 2014), comparando los ejemplares con colecciones de referencia y consultando a especialistas (ver Agradecimientos). Los ejemplares que no se pudieron identificar al nivel de especie fueron considerados como morfoespecies. Todos los especímenes fueron depositados en las colecciones de la Cátedra de Entomología de la Facultad de Humanidades y Ciencias (FHUC, UNL, Santa Fe, Argentina), del Museo Provincial de Ciencias Naturales Florentino Ameghino (MFA, Santa Fe, Argentina) o del Museo Argentino de Ciencias Naturales Bernardino Rivadavia (MACN, Buenos Aires, Argentina).

\section{Rol ecosistémico de los insectos}

Los insectos colectados fueron clasificados en cinco grupos funcionales relevantes para evaluar su rol ecosistémico (modificado de Torretta and Poggio 2013): depredadores (especies que capturan otros artrópodos para alimentar a sus larvas), parasitoides e hiperparasitoides (especies que desarrollan su ciclo larvario en insectos vivos y que matan a su huésped al eclosionar), herbívoros (especies que se alimentan de tejidos de plantas vivas), descomponedores (especies cuyo ciclo larvario se desarrolla en materia orgánica de origen animal o vegetal en descomposición) y polinizadores (especies que visitan flores para colectar polen y néctar para alimentar a sus larvas).

Dado que en muchas especies la dieta del estado adulto difiere de la de sus estados inmaduros, la clasificación se basó en el principal componente de la dieta de cada especie. Para la clasificación se utilizó información disponible en bibliografía (McAlpine et al. 1981; Morrone and Coscarón 1998; Cordo et al. 2004; Triplehorn and Johnson 2005; Fernández and Sharkey 2006; Michener 2007; Claps et al. 2008; Roig-Juñent et al. 2014). Las morfoespecies fueron clasificadas según la información bibliográfica disponible para el taxa superior (género o familia) al que fueron asignadas. En la familia Syrphidae se colectaron individuos adultos exclusivamente y fueron considerados polinizadores (Garibaldi et al. 2011).

\section{Redes de interacciones en taipas}

Con los registros obtenidos de los muestreos de plantas e insectos en las taipas se construyeron matrices con el número total de visitas observadas para cada interacción planta-visitante floral durante todo el período de estudio bajo cada tipo de manejo (EMA y EMC). Las redes de interacción obtenidas se caracterizaron con parámetros de red cualitativos y cuantitativos estimados con el paquete bipartite (Dormann et al. 2008) en la plataforma RStudio (Rstudio Team 2020). Los parámetros cualitativos estimados fueron el número de taxones de plantas e insectos en cada red y el número medio de enlaces (links) por especie. Los parámetros cuantitativos calculados fueron: a) generalidad (número medio ponderado de especies de plantas por especie de visitante), b) conectancia (proporción observada respecto a todas las posibles interacciones ponderadas por la tasa de visita cuantitativa de cada especie; Bersier et al. 2002; Kaiser-Bunbury et al. 2009), y c) superposición de nicho (similitud media en el patrón de interacción entre especies del mismo nivel trófico, con valores $\sim 0$ que indican que no hay un uso común de los nichos y 1 indicando una superposición de nicho perfecta [Dormann et al. 2009]). Los parámetros cuantitativos ponderan las interacciones según su frecuencia, lo que los hace menos sensibles a la intensidad de muestreo y al tamaño de la red.

\section{Análisis de datos}

Con el total de insectos colectados en cada fecha de muestreo se estimaron la riqueza, la abundancia de insectos y la diversidad de especies (o morfoespecies) según el índice de Shannon en cada ambiente: taipas agroecológicas (TA), taipas convencionales (TC), cultivos agroecológicos (CA) y cultivos convencionales (CC) ubicados en 
los dos establecimientos (EMA y EMC). Las diferencias de abundancia y riqueza de especies de insectos entre los dos tipos de manejo (promediando por transectas los datos de cada jornada de muestreo para cada establecimiento EMA; EMC: $n=12$ ) se pusieron a prueba estadísticamente con pruebas no paramétricas de Mann-Whitney (U), ya que la distribución de los datos no cumplía con los supuestos de normalidad. Para el análisis de las diferencias de riqueza y abundancia de los cinco grupos funcionales entre tipos de manejo se realizaron pruebas $t$ de Student luego de corroborar que los residuos eran compatibles con los supuestos de normalidad y homogeneidad de varianzas. Las pruebas estadísticas se realizaron con los paquetes básicos de R (R Core Team 2020) y las estimaciones de diversidad y riqueza se obtuvieron con el paquete vegan 2.5-6 (Oksanen et al. 2019) dentro de la plataforma Rstudio (RStudio Team 2020). Para todas las puesta a prueba de hipótesis se utilizó un nivel de significación de alfa=0.05.

\section{Resultados}

\section{Diversidad de insectos en cultivos}

Se capturaron un total de 1008 insectos pertenecientes a 237especies y morfoespecies, principalmente de los órdenes Hymenoptera (46.8\%), Diptera (19.4\%), Coleoptera (13.1\%) y Hemiptera (9.8\%) (Material Suplementario I). El valor del índice de diversidad de especies estimado fue mayor en el establecimiento bajo manejo agroecológico que en aquel bajo manejo convencional (índices de Shannon: $\mathrm{EMA}=4.46, \mathrm{EMC}=4.35)$. El valor de este índice fue mayor para las taipas de ambos establecimientos $(\mathrm{TA}=4.41, \mathrm{TC}=4.09)$ que para los cultivos (CC=3.61, $C A=3.18)$.

Los valores muestrales de riqueza y abundancia de especies de insectos fueron $71 \%$ y $29 \%$ mayores en EMA que en EMC respectivamente, pero no presentaron diferencia estadísticamente significativa (Tabla 1).

La riqueza y la abundancia de insectos en el EMA fueron en aumento con el tiempo dentro de la temporada 2017-2018, con un descenso recién en el último periodo (febrero). En cambio, en EMC la riqueza de especies se mantuvo estable y la abundancia fue decreciendo levemente con el tiempo. Sólo al inicio de la temporada (noviembre) la riqueza y la abundancia en EMC fueron mayores que en EMA (Figura 2).
Tabla 1. Riqueza de especies y abundancia (promedio y DE) de insectos colectados en dos establecimientos de cultivo de arroz en el centro-este de la provincia de Santa $\mathrm{Fe}$ (Argentina), uno con manejo agroecológico (EMA, $n=12$ transectas promediadas por fechas de visita durante la temporada 2017-2018) y otro con manejo convencional (EMC, $n=12)$. Los valores de P corresponden a pruebas no paramétricas de Mann-Whitney (U).

Table 1. Species richness and abundance (mean and SD) of insects collected from two rice farms in centraleastern Santa Fe province, one under agroecological management (EMA, $n=12$ transects, averaged by four survey dates during the 2017-2018 crop season) and the other under conventional management (EMC, $n=12)$. P values correspond to non-parametric Mann-Whitney (U) tests.

\begin{tabular}{lcccccc}
\hline $\begin{array}{l}\text { Estable- } \\
\text { cimiento }\end{array}$ & \multicolumn{3}{c}{$\begin{array}{c}\text { Riqueza de } \\
\text { especies }\end{array}$} & \multicolumn{3}{c}{ Abundancia } \\
\hline & $\overline{\mathrm{x}}$ & $\mathrm{DE}$ & $\mathrm{P}$ & $\overline{\mathrm{x}}$ & $\mathrm{DE}$ & $\mathrm{P}$ \\
EMA & 9.7 & 7.2 & 0.47 & 25.6 & 26.2 & 0.14 \\
EMC & 7.5 & 5 & & 14.7 & 13.7 & \\
\hline
\end{tabular}

\section{Grupos funcionales}

Depredadores. La riqueza de especies en este grupo fue igual tanto en las TA como en las TC, con 24 especies cada una (Figura 3a), con valores mucho más bajos en el CC (7) y el CA (3), por lo que los establecimientos no difirieron significativamente (Tabla 2). En las TA la abundancia total de este grupo funcional fue $69 \%$ mayor (76 individuos) que en TC (45) y en CC la abundancia de depredadores fue mayor que en CA (16 y 4, respectivamente) (Figura $3 b)$. La abundancia promedio observada en las muestras en EMA fue mayor que en las de EMC, aunque no se encontraron diferencias estadísticamente significativas entre ambos tipos de manejo (Tabla 2). El grupo estuvo principalmente representado por avispas de la familia Vespidae. Las especies Brachygastra lecheguana (Latreille) y Polybia ignobilis (Haliday) presentaron mayor abundancia en el EMA (25 y 17 individuos, respectivamente). En este grupo funcional se colectaron coleópteros pertenecientes a la familia Coccinellidae (Eriopis connexa [Germar], Cycloneda sanguínea [L.]) en ambos tipos de manejo. En cuanto a Diptera, se colectaron 23 individuos de la familia Sciomyzidae en TA, CA y TC.

Parasitoides. La riqueza para este grupo fue $100 \%$ mayor en TA (14) (Figura 3a) que en TC (7), y en CC sólo se capturaron 2 individuos. No se registraron diferencias significativas entre los establecimientos (Tabla 2). En cuanto a la abundancia (Figura 3b), las TA (70) presentaron valores mayores, seguido en orden decreciente por CC y TC (11 y 3, respectivamente). Nosecolectaron parasitoides dentro del CA. Sin embargo, al comparar 
Tabla 2. Análisis entre manejo por grupo funcional de la riqueza de especies y abundancia de cinco grupos funcionales de insectos colectados en establecimientos de cultivo de arroz en el centro-este de la provincia de Santa Fe (Argentina), uno con manejo agroecológico (EMA, $n=12$ transectas promediadas por fechas de visita durante la temporada 2017-2018 y otro con manejo convencional (EMC, $n=12)$. Los valores de P corresponden a pruebas $t$ de Student $\left({ }^{*}: P<0.05\right)$.

Table 2. Analysis between managements per functional group of species richness and abundance of five functional groups of insects collected from two rice farms in central-eastern Santa Fe province (Argentina), one under agroecological management (EMA, $n=12$ transects averaged by survey dates during the 2017-2018 crop season) and the other under conventional management (EMC, $n=12)$. P values correspond to Student's $t$ tests $\left({ }^{*}: P<0.05\right)$.

\begin{tabular}{llcccccc}
\hline & & \multicolumn{3}{c}{ Riqueza } & \multicolumn{5}{c}{ Abundancia } \\
Grupo funcional & Establecimiento & $\overline{\mathrm{X}}$ & $\mathrm{DE}$ & $P$ & $\overline{\mathrm{X}}$ & $\mathrm{DE}$ & $P$ \\
\hline Depredadores & EMA & 5.4 & 1.8 & 0.25 & 16 & 11.2 & 0.17 \\
& EMC & 6.2 & 1.3 & & 12.2 & 1.52 & \\
Parasitoides & EMA & 2.8 & 1.6 & 0.38 & 14 & 12.39 & $0.01^{*}$ \\
& EMC & 1.8 & 0.8 & & 2.8 & 1.48 & \\
Herbívoros & EMA & 14 & 16 & \multirow{2}{*}{0.63} & 35.4 & 33.63 & $0.02^{*}$ \\
Descomponedores & EMC & 14.6 & 10 & & 23.8 & 15.99 & \\
\multirow{3}{*}{ Polinizadores } & EMA & 8.2 & 8.6 & 0.45 & 14.4 & 14.98 & 0.38 \\
& EMC & 5.6 & 4.7 & & 11 & 8.75 & \\
& EMA & 17.4 & 7.7 & 0.77 & 48 & 34.98 & 0.16 \\
\hline
\end{tabular}
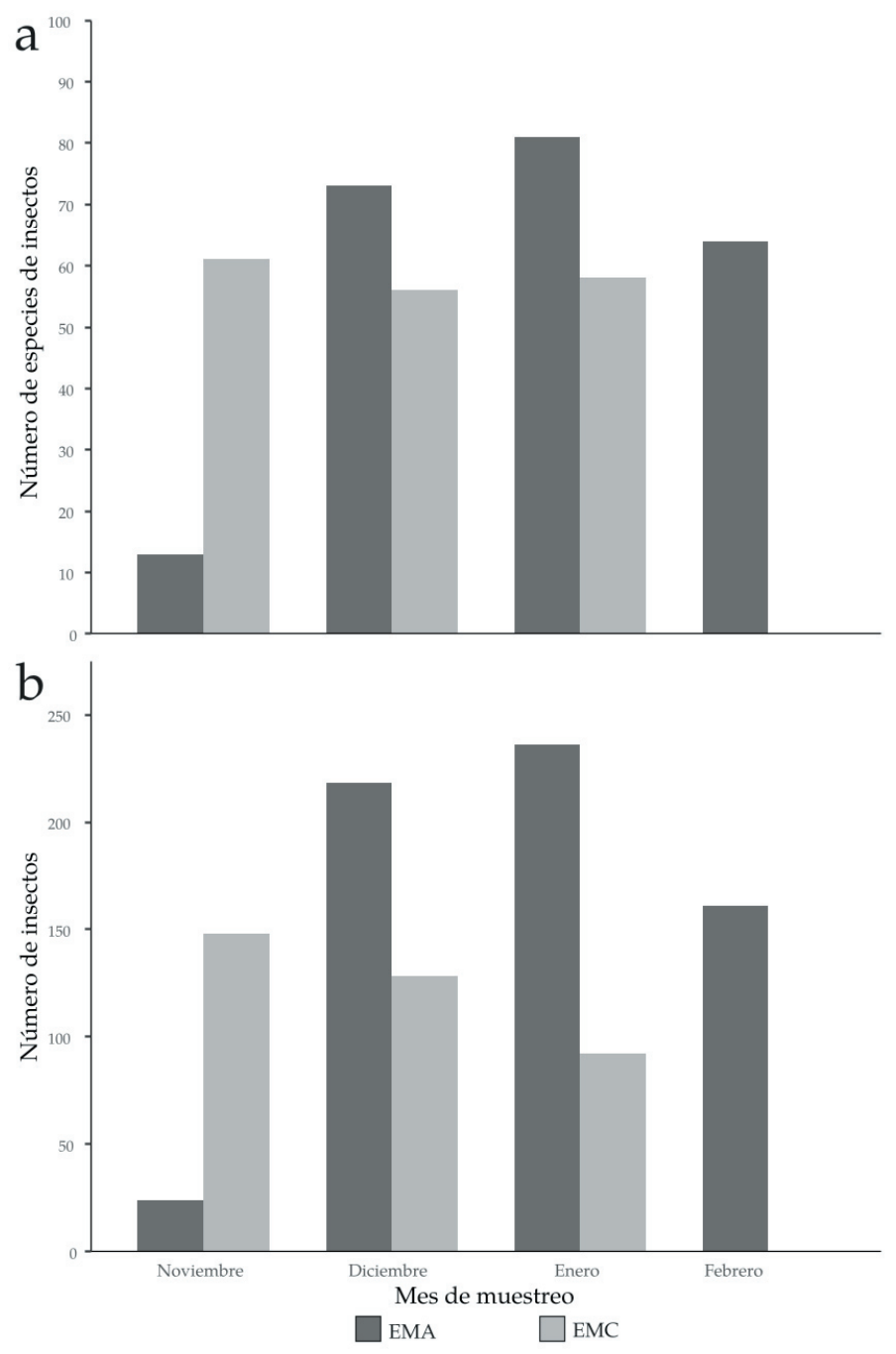

Figura 2. a) Riqueza de especies o morfoespecies y b) abundancia total de insectos por mes de muestreo encontrados en un establecimiento de cultivo de arroz bajo manejo convencional (EMC) y uno bajo manejo agroecológico (EMA) en el centro-este de la provincia de Santa Fe (Argentina). No se relevaron insectos en febrero en EMC.

Figure 2. a) Species or morphospecies richness, and b) total abundance of insects for each sampling month recorded in the farm under conventional management (EMC) and the farm under agroecological management (EMA). Insects were not surveyed during February in EMC. 


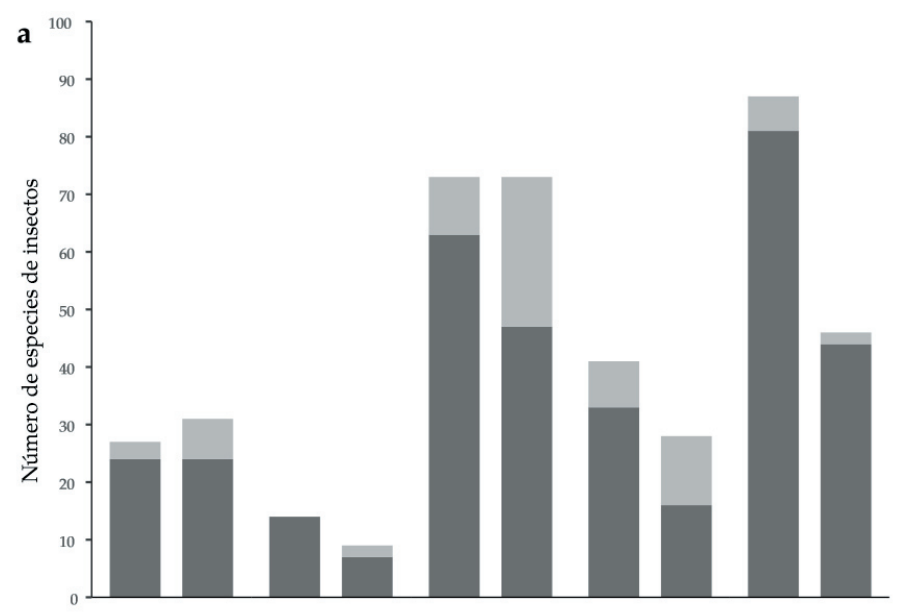

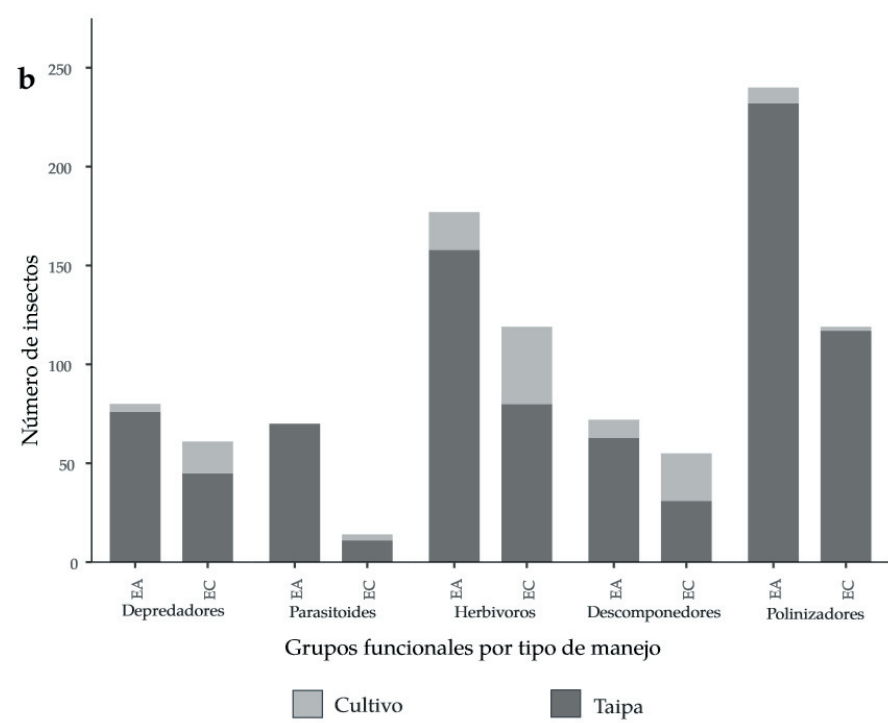

Figura 3. a) Riqueza de especies y b) abundancia en cada grupo funcional de insectos detectados en cultivos de arroz y sus taipas en un establecimiento de cultivo de arroz bajo manejo convencional (EMC) y uno bajo manejo agroecológico (EMA) en el centro-este de la provincia de Santa Fe (Argentina).

Figure 3. a) Species richness and b) abundance in each functional group of insects recorded in rice crops and containment edges (taipas) in a farm under conventional management (EMC) and a farm under agroecological management (EMA) in central-eastern Santa Fe province (Argentina). entre establecimientos, la abundancia en el EMA fue significativamente mayor que en EMC (Tabla 2). De las 11 especies colectadas todas pertenecieron al orden Hymenoptera; la más abundante fue Lysiphlebus testaceipes (Cresson).

Herbívoros. La riqueza para este grupo fue $34 \%$ mayor para la TA (63) (Figura 3a) que para la TC, pero mayor en CC que en CA ( 26 y 10, respectivamente). La riqueza fue similar al comparar los establecimientos (Tabla 2). En cuanto a la abundancia, las TA presentaron un $98 \%$ más (158) de herbívoros (Figura 3b) que las TC (80) siguiendo, en orden decreciente, CC y CA (39 y 19, respectivamente). La abundancia de este grupo fue significativamente mayor en el EMA que en EMC (Tabla 2). El orden Hemiptera presentó 42 especies de herbívoros, entre las que se colectó Oebalus poecilus (Dallas) en CC y CA. También se colectaron especies de coleópteros de las familias Chrysomelidae y Elateridae, y alta abundancia de la familia Tettigonidae (Orthoptera) en ambos establecimientos. Otras taxas consideradas perjudiciales para el cultivo fueron Diabrotica speciosa (Germar), Liorhyssus hyalinus (F.), Ortilia ithra (W. F. Kirby), Aleuas vitticollis (Stål), Dichroplus maculipennis (Blanchard), que sólo se colectaron en CC.

Descomponedores. TA mostró mayor riqueza para este grupo funcional (33) que TC (Figura 3a), mientras que CC presentó mayor riqueza que CA (12 y 8, respectivamente). En cuanto a la abundancia de este grupo funcional (Figura $3 b)$, las TA también presentaron valores mayores (63) que TC (31) y en CC se encontró un $62.5 \%$ (24) más descomponedores que en CA (9). Este grupo de insectos no presentó diferencias significativas en su abundancia ni en su riqueza entre establecimientos (Tabla 2). En total se colectaron 38 especies y morfoespecies de insectos descomponedores, pertenecientes a 10 familias. El orden Diptera presentó mayor riqueza y abundancia (33 y 
59, respectivamente), siendo Sarcophagidae la familia con mayor riqueza registrada dentro de este grupo funcional (18 especies).

Polinizadores. La riqueza en las TA (81) fue $84 \%$ mayor que en TC (44) seguido por el CA y el CC (6 y 2, respectivamente, Figura 3a). En cuanto a la abundancia, las TA presentaron un $98 \%$ más (232) polinizadores (Figura 3b) que en las TC (117), ambos con valores mucho más grandes que en los cultivos CA y CC (8 y 2, respectivamente). Este grupo de insectos no presentó diferencias significativas en su abundancia ni en su riqueza al considerar los establecimientos (Tabla 2). Los polinizadores en su mayoría pertenecieron a la superfamilia Apoidea (57 especies) y Apis mellifera L. fue el polinizador más abundante (41 individuos

a

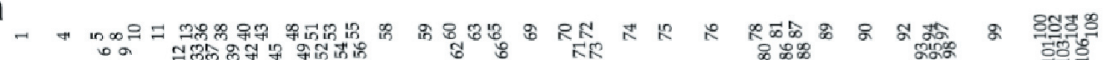
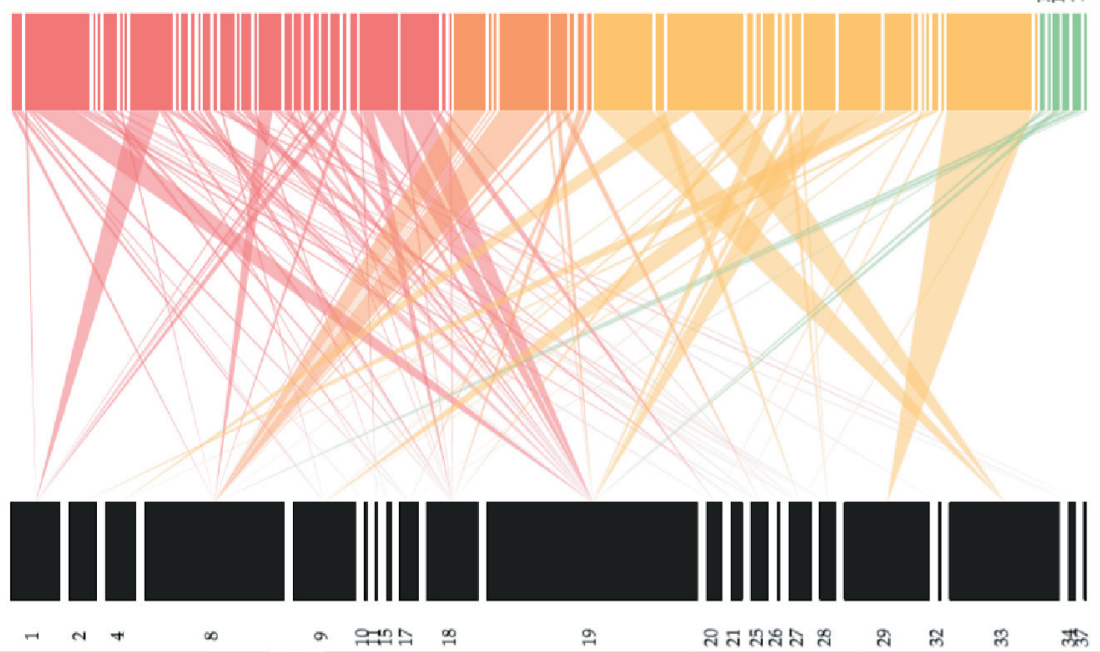

9

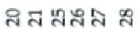

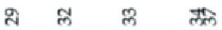

$\mathrm{b}$
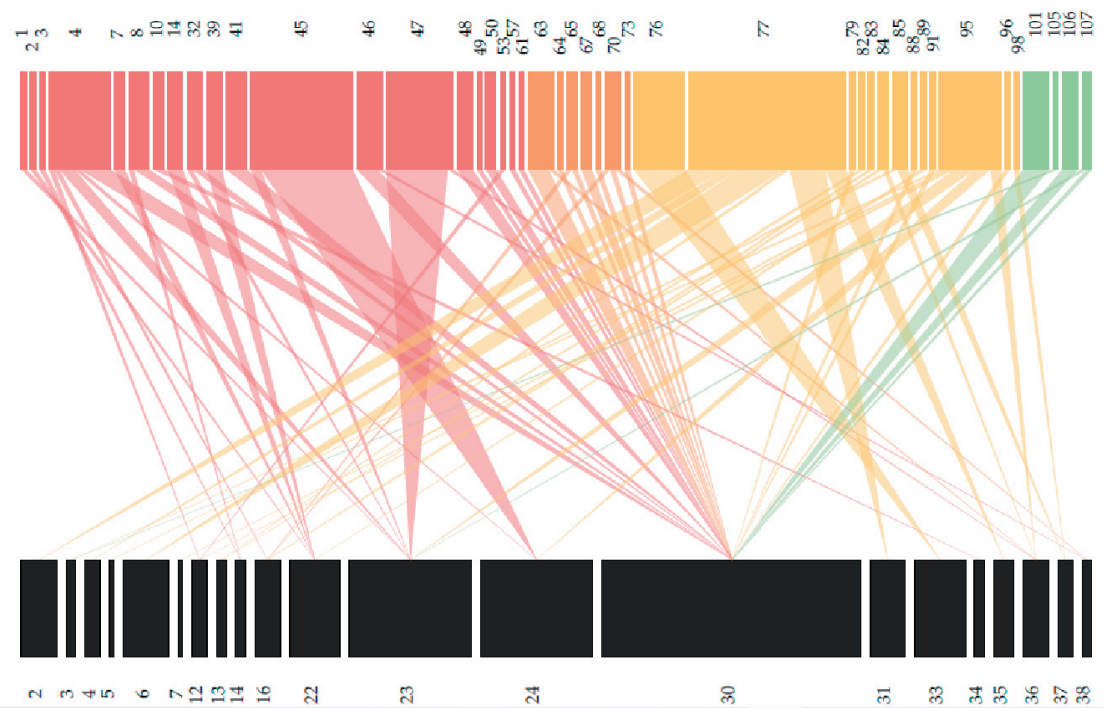

Figura 4. Redes de interacciones mutualistas planta-insecto visitante floral obtenidas a partir de muestreos sobre taipas de dos establecimientos de cultivo de arroz en el centro-este de la provincia de Santa Fe (Argentina). a) Taipa en establecimiento bajo manejo agroecológico (TA). b) Taipa en establecimiento bajo manejo convencional (TC). Los colores en la fila superior indican los grupos funcionales en los que se clasificaron a los insectos (rojo: polinizadores; naranja: depredadores; amarillo: herbívoros; verde: descomponedores). En la fila inferior están las especies de plantas con flor que se identificaron sobre las taipas. La identidad taxonómica de las especies se presenta en los Materiales Suplementarios I y II.

Figure 4. Networks of mutualistic plant-insect floral visitor interactions obtained from sampling on containment edges (taipas) of two rice farms in central-eastern Santa Fe province (Argentina). a) Containment edge under agroecological management (TA). b) Containment edge under conventional management (TC). Colors in the upper row indicate functional groups in which insects were classified (red: pollinators; orange: predators; yellow: herbivores; green: decomposers). Flowering plant species that were identified on the containment edge are in the lower row. Taxonomic identity of species is presented in Supplementary Materials I and II. 
Tabla 3. Métricas de red usadas para caracterizar las redes de interacción planta-visitante floral de las taipas del establecimiento con manejo agroecológico (TA) y las taipas del establecimiento con manejo convencional (TC) del cultivo de arroz en el centro-este de la provincia de Santa Fe (Argentina). Las métricas fueron calculadas para todas las especies. Las redes se presentan en la Figura 4.

Table 3. Metrics used for characterizing networks of the interaction of plant-flower visitors of contention edges (taipas) for the rice farm under agroecological management (TA) and for the farm under conventional management (TC) in central-eastern Santa Fe province (Argentina). Metrics were calculated for all the species. Networks are presented in Figure 4.

\begin{tabular}{lcc}
\hline Métricas & TA & TC \\
\hline Generalidad & 1.67 & 2.42 \\
Conectancia & 0.07 & 0.08 \\
Enlaces por especie & 1.15 & 1.08 \\
Superposición de nicho del nivel superior & 0.17 & 0.28 \\
\hline
\end{tabular}

colectados). Además, se colectaron especies de Dípteros de las familias Syrphidae y Bombyliidae.

\section{Redes de interacciones en bordes}

En TA y TC se registraron un total de 38 especies y morfoespecies de plantas, pertenecientes a 16 familias (Material Suplementario II). En TA se registraron 22 especies vegetales y en TC, 21 especies. Sobre esas plantas se registró un total de 88 especies de insectos ( $\mathrm{TA}=64, \mathrm{TC}=44$ especies). En las TA se registró la mayor parte $(69.4 \%)$ del total de interacciones planta-visitante floral (572). Ludwigia elegans (Cambess.) H. Hara fue la planta en la que se registró mayor número de visitas de insectos (94) (Figura 4). El promedio de enlaces por especie fue mayor en la red construida para TA, mientras que la conectancia de la red, la generalidad y la superposición de nicho del nivel superior fueron mayores en TC (Tabla 3).

En TA se registraron 393 interacciones (Figura $4 a)$ de las cuales $43.8 \%$ correspondieron al grupo funcional herbívoros (174), 39.5\% a polinizadores (157), $13.1 \%$ a depredadores (52) y $3.5 \%$ a descomponedores (14). Mientras tanto, en las TC se registraron 175 interacciones (Figura $4 b$ ) de las cuales el $47.4 \%$ las realizaron los polinizadores (83), 37.7\% correspondieron a herbívoros (66), $8.6 \%$ a depredadores (15), y $6.3 \%$ a descomponedores (11). Los polinizadores interactuaron con 17 especies de plantas de crecimiento espontáneo en las TA y sólo con 8 especies en las TC. No fueron registrados parasitoides en el muestreo de plantas-visitantes florales.

\section{Discusión}

Los resultados obtenidos en este trabajo constituyen un aporte novedoso al conocimiento de la diversidad de insectos presentes en taipas y cultivo de arroz. Si bien existen otros relevamientos de insectos en la provincia de Santa Fe (Montero 2008; Torretta et al. 2009; Dalmazzo 2010; Torretta and Poggio 2013), este sería el primero realizado en arroz, aportando conocimientos de la diversidad, composición y distribución de la comunidad de insectos como así también datos de la oferta floral de la vegetación que crece en taipas y su relación con la entomofauna.

El establecimiento con manejo agroecológico (EMA) presentó mayor diversidad de insectos que el convencional (EMC), lo que demuestra los beneficios de las prácticas agrícolas basadas en biodiversidad para las comunidades de insectos, indicados también en otros estudios realizados en arroz (Pires et al. 2016; Acosta et al. 2017; Silva et al. 2020). La riqueza y la abundancia de especies de insectos en agroecosistemas responde a procesos que actúan a distintas escalas (Molina et al. 2016). En nuestro estudio, la similitud de los valores de riqueza obtenidos para los dos establecimientos podría explicarse por factores que operan a escala de paisaje. Estos factores estarían relacionados con características propias de los sistemas de cultivo de arroz, que incluyen la presencia de espacios donde crece vegetación no cultivada (taipas), lo cual proporciona ambientes más heterogéneos $y$ estables que otros monocultivos (Bambaradeniya 2003; Bambaradeniya and Amerasinghe 2004; Silva et al. 2020). Además, los establecimientos donde se realizó el estudio se encuentran ubicados a 200 m de distancia de remanentes de monte nativo en los que se realiza actividad ganadera. Estos ambientes aportan diversidad al paisaje y podrían estar manteniendo comunidades de insectos ricas en especies que se desplazan hacia los establecimientos productores de arroz (de Souza da Silva et al. 2016; Silva et al. 2020). Los valores de riqueza y abundancia fueron en aumento conforme avanzó el ciclo de cultivo, aunque esta tendencia fue menos marcada en el establecimiento con manejo convencional que utiliza una variedad de arroz de ciclo corto. La mayor abundancia en noviembre en EMC, al inicio de la temporada, podría deberse a la preponderancia de poblaciones de herbívoros, ya que este grupo funcional fue el más abundante en dicho establecimiento. Otros estudios también registraron picos de 
herbívoros durante las etapas vegetativas de arroz (octubre-noviembre) asociados a cultivos con taipas sin vegetación (Acosta et al. 2017). Otro factor que se asocia al aumento del número de herbívoros en producciones convencionales es el uso de insecticidas de amplio espectro que provocan mortandad de insectos depredadores (Ali et al. 2019). En los meses siguientes EMA presentó mayor abundancia que EMC, alcanzando el máximo valor en la fase reproductiva (enero) y decreciendo hacia el final del ciclo (febrero), un patrón coincidente con lo descripto en cultivos de arroz orgánico del sur de Brasil (Acosta et al. 2017).

\section{Insectos y servicios ecosistémicos}

La riqueza de especies por grupo funcional no presentó diferencias significativas entre tipo de manejo. Esto podría explicarse por la influencia de factores ya mencionados (los inherentes a los sistemas de cultivo de arroz porirrigación y presencia de vegetación natural cercana), que a escala de paisaje favorecen la diversidad y la riqueza de insectos. No obstante, esta idea se debería explorar con técnicas de muestreo complementarias a escala de paisaje, que se prolonguen en el tiempo para corroborar este patrón en sucesivos ciclos de cultivo. Otros factores a escala local, inherentes a la práctica de manejo (como la aplicación de insecticidas), podrían estar explicando mejor los valores de abundancia, que en el caso de los parasitoides y herbívoros presentaron diferencia estadísticamente significativa entre manejo.

El análisis por grupo funcional permite detectar potenciales interacciones tróficas que se traducen en servicios ecosistémicos que podría estar brindando la entomofauna (Tscharntke et al. 2005) en cultivos de arroz. Depredadores y parasitoides son considerados proveedores de servicio de regulación natural y control biológico (de Souza da Silva 2016; Ali et al. 2019) de otros insectos herbívoros. En el caso de los depredadores, si bien no se registraron diferencias estadísticamente significativas en la abundancia promedio entre establecimientos, se recolectaron $69 \%$ más individuos en las taipas agroecológicas que en las taipas convencionales. Los parasitoides presentaron diferencia significativa entre manejos, con valores de abundancia mayores en el EMA. La biodiversidad presente en las taipas, favorecida por prácticas agroecológicas, podría explicar la abundancia de estos grupos funcionales. Otros estudios encontraron que la diversidad de parasitoides se asocia a taipas con vegetación, tanto en producción orgánica como convencional (Bambaradeniya and Edirisinghe 2009; Pires et al. 2016; Silva et al. 2020). En nuestro estudio se puede destacar la presencia de E. connexa, considerado un depredador importante de estadios iniciales de Nezara viridula (L.), y de la chinche del arroz $O$. poecilus, ambas perjudiciales para este cultivo (Gallo et al. 2002). La familia Sciomyzidae (Diptera), conocidas comúnmente como 'moscas de pantano', está formada casi exclusivamente por depredadores obligados de moluscos (Chapman et al. 2012) que resultan relevantes como agente de biocontrol de gasterópodos (Marinoni and Knutson 2010; Kirst et al. 2015). En este trabajo se registraron dos géneros de esta familia (Sepedonea sp.1 y Protodictya sp.1) que pueden considerarse controladores de Pomacea canaliculata (L.), un problema importante en los cultivos que permanecen inundados (Kruger and Burdyn 2015).

Si bien el EMA presentó mayor abundancia (177) de herbívoros, su zona de cultivo (CA) representó sólo el 10.7\% del total. En cambio, CC presentó casi un tercio (32.8\%) del total de herbívoros colectados en el EMC. La presencia de poblaciones numerosas de insectos herbívoros encontradas en las TA podría contribuir a mantener poblaciones viables de depredadores y parasitoides en este establecimiento, disminuyendo la frecuencia de interacciones de interferencia intra-gremio. Se ha indicado que este tipo de interacciones (i.e., canibalismo, depredación y parasitismo) son características de las comunidades de enemigos naturales polífagos (Lang 2003) y resultan relevantes para el control biológico (Rosenheim et al.1995; Brodeur and Boivin 2006).

Los valores muestrales promedio de riqueza y abundancia de descomponedores y polinizadores fueron mayores en EMA. El grupo funcional polinizadores fue el más abundante en los dos establecimientos, en coincidencia con reportes previos de su alta diversidad en cultivos de arroz por irrigación (Bambaradeniya and Edirisinghe 2009), en particular en las taipas. Aunque el arroz no requiere agentes de polinización, consideramos importante mantener ambientes que resguarden poblaciones de estas especies para asegurar la reproducción de muchas especies vegetales no cultivadas que crecen en las taipas y sostienen a los otros grupos funcionales. 


\section{Redes de interacción}

Si bien el efecto de prácticas agrícolas amigables con el ambiente (producción orgánica) sobre la riqueza y diversidad de insectos en cultivos de arroz ya ha sido estudiado (Bambaradeniya and Edirisinghe 2009; Acosta et al. 2017; Ali et al. 2019; Silva et al. 2020), el estudio de las interacciones plantavisitante floral desde la perspectiva de redes en taipas es un aporte novedoso que puede ayudar a comprender la respuesta de los organismos y sus interacciones a las prácticas de manejo agrícola (Memmott 2009; Power and Stout 2011). El número de interacciones en redes mutualistas fue identificado como un índice de importancia para la caracterización de su estructura (Okuyama and Holland 2008). En el presente estudio, el número de interacciones planta-visitante floral fue $30.6 \%$ mayor en EMA que en EMC aun cuando no se registraron diferencias entre establecimientos ni en la riqueza promedio de especies vegetales ni en la de insectos (Material Suplementario I y II). El mayor número de interacciones planta-visitante floral registrado en EMA podría atribuirse a que es un ambiente libre de insecticidas (Attademo et al. 2014), una de las causas de la disminución de polinizadores en agroecosistemas (Potts et al. 2010). Las prácticas agroecológicas serían favorables para la conservación de interacciones plantavisitante floral, ecológicamente importantes para sustentar la biodiversidad, como ya se mostró en viñedos (Kehinde and Samways 2014).

Otros parámetros de descripción de las redes como la conectancia, los enlaces por especie y la superposición de nicho del nivel superior (insectos), fueron similares entre los establecimientos comparados. Estos parámetros suelen estar relacionados, ya que frecuentemente están influenciados por procesos biológicos similares (Vázquez et al. 2007, 2009; Okuyama and Holland 2008). De cualquier modo, sería necesario compararlos con un número mayor de redes para comprender cómo varían con la complejidad de las redes de interacción en taipas bajo diferente tipo de manejo.

Por otro lado, la construcción de redes posibilitó caracterizar la importancia de la vegetación como recurso para cuatro grupos funcionales de insectos. Se detectó que las flores de Eryngium sp.1 en TA y de Sphaeralcea bonariensis (Cav.) Griseb en TC fueron las más utilizadas por depredadores para complementar su dieta, mientras que $L$. elegans (94 visitas) y Eryngium sp.1 (62 visitas), especies sólo registradas en TA, fueron las más visitadas por la comunidad de insectos en general.

\section{Taipas y vegetación no cultivada}

Los resultados de este trabajo muestran la importancia de la vegetación no cultivada que crece en las taipas, inclusive en los establecimientos con manejo convencional. A diferencia de la mayoría de las plantas con flores, el arroz carece de recurso nectarífero para complementar la dieta de enemigos naturales de plagas. La vegetación de las taipas aporta refugio, polen, néctar, presas y huéspedes alternativos (Bambaradeniya and Edirisinghe 2009; Gurr et al. 2011), y promueve la biodiversidad y la complejidad del agroecosistema (Molina et al. 2016, 2019). Desde esta base conceptual ha sido evaluada. Los estudios de implantación de especies vegetales con flores ricas en polen y néctar sobre taipas demostraron que aumentan los agentes de control biológico (depredadores y parasitoides), se reduce el número de insectos plaga y el daño causado al cultivo, y se mantiene el rendimiento del cultivo, en comparación con prácticas que utilizan insecticidas sin manejo de taipas (Gurr et al. 2004; Ali et al. 2019). La implementación de futuros planes de manejo de vegetación en taipas debería considerar los resultados de este trabajo (i.e., abundancia y composición de grupos funcionales de insectos, oferta floral e interacciones planta-visitante floral) para asegurar recursos suficientes a los insectos considerados benéficos en estos establecimientos arroceros. Las prácticas agroecológicas, basadas en biodiversidad sin aplicación de insecticidas, favorecerían la presencia de comunidades de insectos que proveen servicios ecosistémicos (control de plagas y polinización) en sistemas de producción de alimentos más seguros para el ambiente y para la salud humana (Ali et al. 2019; Dainese et al. 2019).

Agradecimientos. A la Cooperativa de trabajo "El progreso" y la Coop. Arrocera "Villa Elisa" que permitieron realizar este estudio en sus campos. Al Dr. G. Marino por su colaboración en la determinación de plantas y a los Dres. P. Mulieri, L. Patitucci y J. Frana por la determinación de insectos. Al editor Dr. F. Milesi y dos revisores anónimos por sus aportes al manuscrito. Este estudio fue financiado por proyectos CAI+D Orientado 
2016 ("Importancia de los bordes de campo de arroz orgánico en el rol de los vertebrados en el control biológico de plagas") y CAI+D Joven 2016 (Cod: 50020150100073LI) de la Universidad Nacional del Litoral. Carla Ghiglione agradece a la Universidad Nacional del Litoral por otorgarle una Cientibeca para la realización de este trabajo.

\section{ReFERENCIAS}

Acosta, L. G., S. M. Jahnke, L. R. Redaelli, and P. R. S. Pires. 2017. Insect diversity in organic rice fields under two management systems of levees vegetation. Brazilian Journal of Biology 77:731-744. https://doi.org/10.1590/1519 6984.19615

Ali, M. P., M. N. Bari, S. S. Haque, M. M. M. Kabir, S. Afrin, F. Nowrin, M. S. Islam, and D. A. Landis. 2019. Establishing next-generation pest control services in rice fields: Eco-agriculture. Scientific Reports 9:1-9. https://doi.org/10.1038 441598-019-46688-6.

Altieri, M. A., and C. I. Nicholls. 2007. Conversión agroecológica de sistemas convencionales de producción: teoría, estrategias y evaluación. Revista Ecosistemas 16:3-12.

Attademo, A. M., P. M. Peltzer, R. C. Lajmanovich, M. Cabagna, and G. Fiorenza. 2007. Plasma B-esterase and glutathione S-transferase activity in the toad Chaunus schneideri (Amphibia, Anura) inhabiting rice agroecosystems of Argentina. Ecotoxicology 16:533-539. https://doi.org/10.1007/s10646-007-0154-0.

Attademo, A. M., P. M. Peltzer, and R. C. Lajmanovich. 2015. Biochemical changes in certain enzymes of Lysapsus limellium (Anura: Hylidae) exposed to chlorpyrifos. Ecotoxicology and Environmental Safety 113:287-294. https: //doi.org/10.1016/i.ecoenv.2014.12.021.

Attademo, A. M., R. Lorenzón, P. Peltzer, and R. Lajmanovich. 2018. Diversity of Anurans in rice fields under organic and conventional management in Santa Fe Province, Argentina. Herpetological Review 49:632-635.

Attademo, A. M., P. M. Peltzer, R. C. Lajmanovich, M. C. Cabagna-Zenklusen, C. M. Junges, and A Basso. 2014. Biological endpoints, enzyme activities, and blood cell parameters in two anuran tadpole species in rice agroecosystems of mid-eastern Argentina. Environmental Monitoring and Assessment 186:635-649. https://doi.org/10.1007/s10661013-3404-z.

Bambaradeniya, C. 2003. Traditional home garden and rice agro-ecosystems in Sri Lanka: An integrated managed landscape that sustains a rich biodiversity. Pp. 18-19 in Proc International Symp Perspectives of the Biodiversity Research in the Western Pacific and Asia in the 21st Century. Kyoto, Japan.

Bambaradeniya, C. N. B., and F. P. Amerasinghe. 2004. Biodiversity associated with the rice field agroecosystem in Asian countries: a brief review. IWMI, Colombo, Sri Lanka. https://doi.org/10.1023/B:BIOC.0000029331.92656.de.

Bambaradeniya, C. N. B., and J. P. Edirisinghe. 2009. Composition, structure and dynamics of arthropod communities in a rice agro-ecosystem. Ceylon Journal of Science (biological Sciences) 37:23-48. https://doi.org/10.4038/ cjsbs.v37i1.494.

Bersier, L. F. C. Banašek-Richter, and M. F. Cattin. 2002. Ouantitative descriptors of food-web matrices. Ecology 83: 2394-2407. https://doi.org/10.1890/0012-9658(2002)083[2394:ODOFWM]2.0.CO;2]

Brodeur, J., and G. Boivin. 2006. Trophic and Guild Interactions in Biological Control. Springer, New York, USA. https: //doi.org/10.1007/1-4020-4767-3.

Burkart, R. N., O. Bárbaro, R. O. Sánchez, and D. A. Gomez. 1999. Ecorregiones de la Argentina. Administración de Parques Nacionales y Secretaria de Recursos Naturales y Desarrollo Sustentable, Buenos Aires, Argentina.

Chacoff, N. P., D. P. Vázquez, S. B. Lomáscolo, E. L. Stevani, J. Dorado, and B. Padrón. 2012. Evaluating sampling completeness in a desert plant-pollinator network. Journal of Animal Ecology 81:190-200. https://doi.org/10.1111 $.1365-2656.2011 .01883 . x$.

Chapin, F. S. III., E. S. Zavaleta, V. T. Eviner, R. L. Naylor, P. M. Vitousek, H. L. Reynolds, D. U. Hooper, S. Lavorel, O. E. Sala, S. E. Hobbie, M. C. Mack., and S. Díaz. 2000. Consequences of changing biodiversity. Nature 405:234-242. https://doi.org/10.1038/35012241.

Chapman, E. G., A. A. Przhiboro, J. D. Harwood, B. A. Foote, and W. R. Hoeh. 2012. Widespread and persistent invasions of terrestrial habitats coincident with larval feeding behavior transitions during snail-killing fly evolution (Diptera: Sciomyzidae). BMC Evolutionary Biology 12:175-197. https://doi.org/10.1186/1471-2148-12-175.

Claps, L. E, G. Debandi, and S. Roig-Juñet. 2008. Biodiversidad de Artrópodos Argentinos Volumen 2. Editorial Sociedad Entomológica Argentina, Mendoza, Argentina.

Cordo, H. A., G. Logarzo, K. Braun, and O. Di Iorio. 2004. Catálogo de los insectos fitófagos de la Argentina y sus plantas asociadas. Editorial Sociedad Entomológica Argentina, Buenos Aires, Argentina.

Dainese, M., E. A. Martin, M. A. Aizen, M. Albrecht, I. Bartomeus, R. Bommarco, L. G. Carvalheiro, R. Chaplin-kramer, V. Gagic, L. A. Garibaldi, J. Ghazoul, H. Grab, M. Jonsson, D. S. Karp, D. K. Letourneau, L. Marini, K. Poveda, R. Rader, H. G. Smith, M. B. Takada, H. Taki, G. Tamburini, and M. Tschumi. 2019. A global synthesis reveals biodiversitymediated benefits for crop production. Science Advances 5:1-14. http://dx.doi.org/10.1126/sciadv.aax0121.

Dalmazzo, M. 2010. Diversidad y aspectos biológicos de abejas silvestres de un ambiente urbano y otro natural de la región central de Santa Fe, Argentina. Rev. Sociedad Entomológica Argentina 69:33-44.

de Remes Lenicov, A. M. M., R. Mariani, A. Maciá, A. Toledo, M. E. Brentassi, M. F. Rossi Batiz, M. I. Catalano, and S. Paradell. 2014. Diversity of planthoppers (Hemiptera: Fulgoromorpha) in rice associated with weeds in Argentina. Studies on Neotropical Fauna and Environment 49:95-105. https://doi.org/10.1080/01650521.2014.925683. 
de Souza da Silva, G., S. Mundstock Jahnke, and M. L. González Ferreira. 2016. Hymenoptera parasitoids in protected area of Atlantic Forest biomes and organic rice field: compared assemblages. Revista Colombiana de Entomología 42:110-117. https://doi.org/10.25100/socolen.v42i2.6680.

Dormann, C. F., B. Gruber, and J. Fründ. 2008. Introducing the bipartite package: analysing ecological networks. R News 8: 8-11.

Dormann, C. F., J. Fründ, N. Blüthgen, and B. Gruber. 2009. Indices, graphs and null models: analyzing bipartite ecological networks. The Open Ecology Journal 2:7-24. https://doi.org/10.2174/1874213000902010007.

Duré, M. I., A. I. Kehr, E. F. Schaefer, and F. Marangoni. 2008. Diversity of amphibians in rice fields from northeastern Argentina. Interciencia 33:523-527.

Edirisinghe, J. P., and C. N. Bambaradeniya. 2010. Rice fields: an ecosystem rich in biodiversity. Journal of the National Science Foundation of Sri Lanka 34:57-59. https://doi.org/10.4038/jnsfsr.v34i2.2084.

Fernández, F., and M. J. Sharkey. 2006. Introducción a los Hymenoptera de la Región Neotropical. Sociedad Colombiana de Entomología y Universidad Nacional de Colombia, Bogotá, Colombia.

Fisher, B., and R. K. Turner. 2008. Ecosystem services: classification for valuation. Biological Conservation 141:11671169. https://doi.org/10.1016/j.biocon.2008.02.019

Gallo, D., O. Nakano, S. Silveira Neto, R. P. L., Carvalho, G. C., Baptista, E. Berti-Filho, J. R. P. Parra, R. A Zucchi, S. B. Alves, J. D. Vendramim, L. C. Marchini, J. R. S. Lopes, and C. Omoto. 2002. Entomología agrícola. FEALQ, Piracicaba, São Paulo, Brasil.

Garibaldi, L. A., I. S.'Dewenter, C. Kremen, J. M. Morales, R. Bommarco, S. A. Cunningham, L. Carvalheiro, N. P. Chacoff, J. H. Dudenhöffer, S. S. Greenleaf, A. Holzschuh, R. I. Isaacs, K. Krewenka, Y. Mandelik, M. M. Mayfield, L. A. Morandin, S. G. Potts, T. H. Ricketts, H. Szentgyörgyi, F. V. Blandina, C. Westphal, R. Winfree, and A. M. Klein. 2011. Stability of pollination services decreases with isolation from natural areas despite honey bee visits. Ecology Letters 14:1062-1072. https://doi.org/10.1111/j.1461-0248.2011.01669.x.

Gurr, G. M., S. D. Wratten, and M. A. Altieri. 2004. Ecological engineering for pest management: Advances in habitat manipulation for arthropods. CSIRO Publishing, Melbourne, Australia. https://doi.org/10.1071/9780643098411.

Gurr, G. M., J. Liu, D. M. Read, J. L. A. Catindig, J. A. Cheng, L. P. Lan, and K. L. Heong. 2011. Parasitoids of Asian rice planthopper (Hemiptera: Delphacidae) pests and prospects for enhancing biological control by ecological engineering. Annals of Applied Biology 158:149-176. https://doi.org/10.1111/j.1744-7348.2010.00455.x.

IPBES-7. 2019. Plenary of the Intergovernmental Science-Policy Platform on Biodiversity and Ecosystem Services. Paris, France.

Kaiser-Bunbury, C. N., J. Memmott, and C. B. Müller. 2009. Community structure of pollination webs of Mauritian heathland habitats. Perspectives in Plant Ecology, Evolution and Systematics 11:241-254. https://doi.org/10.1016 ippees.2009.04.001.

Kehinde, T., and M. J. Samways. 2014. Insect-flower interactions: Network structure in organic versus conventional vineyards. Animal Conservation 17:401-409. https://doi.org/10.1111/acv.12118.

Kirst, F. D., L. Marinoni, and R. F. Krüger. 2015. New_distribution records for Sciomyzidae species (Insecta, Diptera) from Rio Grande do Sul, Brazil. Check List 11:1552. https://doi.org/10.15560/11.1.1552

Kremen, C., N. M. Williams, R. L. Bugg, J. P. Fay, and R. W. Thorp. 2004. The area requirements of an ecosystem service: crop pollination by native bee communities in California. Ecology Letters 7:1109-1119. https://doi.org/10.1111/j.14610248.2004.00662.x

Kruger, R. D., and L. Burdyn. 2015. Guía para la identificación de plagas del cultivo del arroz (Oryza Sativa L.). INTA, Corrientes, Argentina.

Lang, A. 2003. Intraguild interference and biocontrol effects of generalist predators in a winter wheat field. Oecologia 134:144-153. https://doi.org/10.1007/s00442-002-1091-5

Le Féon, V., S. L. Poggio, J. P. Torretta, C. Bertrand, G. A. R. Molina, F. Burel, J. Baudry, and C. M. Ghersa. 2016. Diversity and life-history traits of wild bees (Insecta: Hymenoptera) in intensive agricultural landscapes in the Rolling Pampa, Argentina. Journal of Natural History 50:1175-1196. https://doi.org/10.1080/00222933.2015.1113315.

Lindgren, J., R. Lindborg, and S. A. Cousins. 2018. Local conditions in small habitats and surrounding landscape are important for pollination services, biological pest control and seed predation. Agriculture, Ecosystems and Environment 251:107-113. https://doi.org/10.1016/i.agee.2017.09.025.

López-Lanús, B., P. Grilli, E. Coconier, A. Di Giacomo, R. Banchs, and G. Marino. 2010. Ecología del Charlatán y su estado actual en la Provincia de Santa Fe, Argentina. Aves Argentinas/Asociación Ornitológica del Plata, Buenos Aires, Argentina.

Lorenzón, R., E. J. León, M. Juani, A. H. Beltzer, P. M. Peltzer, R. C. Lajmanovich, and A. M. Attademo. 2020. Can agroecological management increase functional diversity of birds in rice fields? Revista de Biología Tropical 68:873883. https://doi.org/10.15517/rbt.v68i3.39261.

Marinoni, L., and L. V. Knutson. 2010. Sciomyzidae. In Brown, B. V., A. Borkent, J. M. Cumming, D. M. Wood, N. E. Woodley and M. A Zumbado (eds.). Manual of Central American Diptera. Vol. 2. NRC Research Press. Ottawa, Canadá.

Marrero, H. J., J. P. Torretta, and D. Medan. 2014. Effect of land use intensification on specialization in plant-floral visitor interaction networks in the Pampas of Argentina. Agriculture, Ecosystems and Environment 188:63-71. https: /doi.org/10.1016/j.agee.2014.02.017.

McAlpine, J. F., B. V. Peterson, G. E. Shewell, H. J. Teskey, J. R. Vockeroth and D. M. Wood. 1981. Manual of Neartic 
Diptera. Research Branch Monograph 27, Ottawa, Canada.

Medan, D., J. P. Torretta, K. Hodara, E. B. de la Fuente, and N. H. Montaldo. 2011. Effects of agriculture expansion and intensification on the vertebrate and invertebrate diversity in the Pampas of Argentina. Biodiversity and Conservation 20:3077-3100. https://doi.org/10.1007/s10531-011-0118-9.

Memmott, J. 2009. Food webs: a ladder for picking strawberries or a practical tool for practical problems? Philos Trans Royal Society B: Biological Sciences 364:1693-1699. https://doi.org/10.1098/rstb.2008.0253

Michener, C. D. 2007. The bees of the world. Johns Hopkins University Press. Baltimore, Maryland, USA.

Molina, G. A., S. L. Poggio, and C. M. Ghersa. 2014. Epigeal arthropod communities in intensively farmed landscapes: effects of land use mosaics, neighbourhood heterogeneity, and field position. Agriculture, Ecosystems and Environment 192:135-143. https://doi.org/10.1016/j.agee.2014.04.013.

Molina, G. A. R., S. L. Poggio, and C. M. Ghersa. 2016. Structural complexity of arthropod guilds is affected by the agriculturallandscape heterogeneity generated by fencerows. Annals of Applied Biology 168:173-184. https://doi.org/ 10.1111/aab.12253

Molina, G. A., S. L. Poggio, and C. M. Ghersa. 2019. Parasitoid diversity and parasitism rates in Pampean agricultural mosaics are enhanced by landscape heterogeneity. Insect Conservation and Diversity 12:309-320. https://doi.org, 10.1111 /icad.12342

Montero, G. A. 2008. Comunidades de artrópodos en vegetación de áreas no cultivadas del sudeste de Santa Fe. MSc Tesis. Maestría en Manejo y Conservación de Recursos Naturales. Facultad de Ciencias Agrarias. Universidad Nacional de Rosario. Zavalla, Santa Fe, Argentina.

Morrone, J. J., and S. Coscarón. 1998. Biodiversidad de artrópodos argentinos: una perspectiva biotaxonómica. Ediciones SUR, La Plata, Buenos Aires, Argentina.

Öckinger, E., and H. G. Smith. 2007. Semi-natural grasslands as population sources for pollinating insects in agricultural landscapes. Journal of Applied Ecology 44:50-59. https://doi.org/10.1111/j.1365-2664.2006.01250.x.

Oksanen, J., F. G. Blanchet, M. Friendly, R. Kindt, P. Legendre, D. McGlinn, P. R Minchin, R. B. O'Hara, G. L. Simpson, P. Solymos, M. Henry, H. Stevens, E. Szoecs, and H. Wagner. 2019. Vegan: Community Ecology Package. R package version 2.5.4.

Okuyama, T., and J. N. Holland. 2008. Network structural properties mediate the stability of mutualistic communities. Ecology Letters 11:208-216. https://doi.org/10.1111/j.1461-0248.2007.01137.x

Pinciroli, M., N. Ponzio, and S. Raquel. 2015. El arroz: alimento de millones. Un texto sencillo para alumnos de las carreras vinculadas a las Ciencias Agrarias y Tecnología de los Alimentos. Universidad Nacional del Centro de la Provincia de Buenos Aires. Tandil, Buenos Aires, Argentina.

Pires, P. R., S., M. Jahnke, and L. R. Redaelli. 2016. Influence of the vegetation management of the leeves in irrigated rice organic in diversity of Hymenoptera parasitoids. Brazilian Journal of Biology 76:774-781. https://doi.org/10.1590/ 1519-6984.06215.

Poggio, S. L., E. J. Chaneton, and C. M. Ghersa. 2010. Landscape complexity differentially affects alpha, beta, and gamma diversities of plants occurring in fencerows and crop fields. Biological Conservation 143:2477-2486. hittps. /doi.org/10.1016/j.biocon.2010.06.014.

Poggio, S. L., E. J. Chaneton, and C. M. Ghersa. 2013. The arable plant diversity of intensively managed farmland: Effects of field position and crop type at local and landscape scales. Agriculture, Ecosystems and Environment 166: 55-64. https://doi.org/10.1016/i.agee.2012.01.013.

Potts, S. G., J. C. Biesmeijer, C. Kremen, P. Neumann, O. Schweiger, and W. E. Kunin. 2010. Global pollinator declines: trends, impacts and drivers. Trends in Ecology and Evolution 25:345-353. https://doi.org/10.1016/j.tree.2010.01.007.

Power, E. F., and J.C. Stout. 2011. Organic dairy farming: impacts on insect-flower interaction networks and pollination. Journal of Applied Ecology 48:561-569. https://doi.org/10.1111/j.1365-2664.2010.01949.x

R Core Team. 2020. R: A language and environment for statistical computing. R Foundation for Statistical Computing, Vienna, Austria. URL: R-project.org.

Roig-Juñent, S., L. E. Claps, and J. J. Morrone. 2014. Biodiversidad de Artrópodos Argentinos. INSUE- Universidad Nacional de Tucumán, San Miguel de Tucumán, Tucumán, Argentina.

Romero, A., M. F. Potter, and K. F. Haynes. 2009. Evaluation of piperonyl butoxide as a deltamethrin synergist for pyrethroid-resistant bed bugs. Journal of Economic Entomology 102:2310-2315. https://doi.org/10.1603 029.102 .0637$.

Rosenheim, J. A., H. K. Kaya, L. E. Ehler, J. J. Marois, and B. A.Jaffe. 1995. Intraguild predation among biological-control agents: theory and evidence. Biological Control 5:303-335. https://doi.org/10.1006/bcon.1995.1038.

RStudio Team. 2020. RStudio: Integrated Development for R. RStudio, PBC, Boston, MA. URL rstudio.com.

Sáez, A., M. Sabatino, and M. Aizen. 2014. La diversidad floral del borde afecta la riqueza y abundancia de visitantes florales nativos en cultivos de girasol. Ecología Austral 24:94-102. https://doi.org/10.25260/EA.14.24.1.0.41.

SEA. 2017. Informe $\mathrm{n}^{\circ}$ 337. Sistema de Estimaciones Agrícolas, Instituto de Investigaciones Científicas y Técnicas (I.D.I.C.Y.T.). Gobierno de la provincia de Santa Fe, Santa Fe, Argentina. URL: tinyurl.com/t7hvyy4z.

SIIA. 2019. Sistema Integrado de Información Agropecuaria, Ministerio de Agricultura, Ganadería y Pesca de la Nación Argentina. URL: siia.gov.an.

Silva, G. S., S. M. Jahnke, and N. F. Johnson. 2020. Riparian forest fragments in rice fields under different management: differences on hymenopteran parasitoids diversity. Brazilian Journal of Biology 80:122 132. https://doi.org/10.1590/ 1519-6984.194760. 
Torretta, J. P., F. Navarro, and D. Medan. 2009. Visitantes florales nocturnos del girasol (Helianthus annuus, Asterales: Asteraceae) en la Argentina. Revista de la Sociedad Entomológica Argentina 68:339-350.

Torretta, J. P., and S. L. Poggio. 2013. Species diversity of entomophilous plants and flower-visiting insects is sustained in the field margins of sunflower crops. Journal of Natural History 47:139-165. https://doi.org/10.1080 00222933.2012 .742162

Triplehorn, C. A., and N. F. Johnson. 2005. Borror and delong's introduction to the study of insects. Brooks. Cole, Belmont, California, USA.

Tscharntke, T., A. M. Klein, A. Kruess, I. Steffan-Dewenter, and C. Thies. 2005. Landscape perspectives on agricultural intensification and biodiversity-ecosystem service management. Ecology Letters 8:857-874. https://doi.org/10.1111 1461-0248.2005.00782.x.

Tylianakis, J. M., T. Tscharntke, and O. T. Lewis. 2007. Habitat modification alters the structure of tropical host-parasitoid food webs. Nature 445:202-205. https://doi.org/10.1038/nature05429.

Vázquez, D. P., C. J. Melián, N. M. Williams, N. Blüthgen, B. R. Krasnov, and R. Poulin. 2007. Species abundance and asymmetric interaction strength in ecological networks. Oikos 116:1120-1127. https://doi.org/10.1111/j.0030$1299.2007 .15828 . x$

Vázquez, D. P., N. Blüthgen, L. Cagnolo, and N. P. Chacoff. 2009. Uniting pattern and process in plant-animal mutualistic networks: a review. Annals of Botany 103:1445-1457. https://doi.org/10.1093/aob/mcp057. 